\title{
Le stress au travail chez les enseignants des collèges en Tunisie
}

\section{Alma Hafsi ${ }^{1}$, Noëlle Lallemand2, Valérie Cohen-Scali²}

L'objectif de l'étude est d'identifier les situations de travail les plus génératrices de stress professionnel chez des enseignants des collèges en Tunisie. Le cadre théorique mobilise les modèles du stress psychologique en milieu professionnel, en particulier celui de Karasek. Nous avons émis l'hypothèse que les conditions de travail des enseignants des collèges d'enseignement général engendreraient un stress professionnel. Cette hypothèse a été testée en administrant le JCQ de Karasek à 351 enseignants des trois types de collèges existants. Les résultats montrent que $18 \%$ d'entre eux s'estiment stressés. Toutefois, les enseignants des collèges professionnels sont moins exposés au stress que les autres, en raison d'une forte latitude décisionnelle et de contrôle dans la réalisation de leurs tâches.

\section{Introduction}

Le contexte tunisien de l'enseignement secondaire Depuis l'indépendance jusqu'à nos jours, La Tunisie met régulièrement en œuvre des mutations dans le système éducatif. Dès 1956, l'État s'est attribué pour mission l'édification d'une société moderne, aspirant à sortir le pays de l'analphabétisme (1979). Cinquante ans plus tard, de nombreuses réformes successives sont toujours en train de relever le défi de la modernisation du système éducatif. La réforme de 1991 figurait parmi les plus innovantes. Elle instituait un enseignement de base d'une durée de neuf années de scolarité, soit un premier cycle d'étude primaire de six années et un second cycle de trois ans d'enseignement préparatoire au collège (Bouttemont, 2002). En 2007-2008, d'autres mesures ont été prises, visant la mise en place d'écoles préparatoires professionnelles et d'écoles préparatoires pilotes. Cette réforme tendait à redéfinir le rôle et les finalités du système éducatif en proposant une nouvelle configuration des établissements scolaires de l'enseignement de base (Loi n91-65 du 20 juillet 1991).

Généralement, ces réformes ont conduit le personnel enseignant à exercer dans un climat d'incertitude et d'instabilité engendrant un sentiment 
d'insécurité. Ainsi, ces changements sont l'objet de discordes entre la prescription et l'application des nouvelles orientations (Diet, 2001). Comme le rappelle Ennaifar (2009), l'implantation de nouvelles réformes n'implique pas seulement l'innovation pédagogique mais aussi l'innovation dans le management des établissements au plan de la vie scolaire, la gestion participative, les conseils d'établissement, l'implication de la communauté éducative et des parents. Les nouveautés introduites par les réformes sont donc susceptibles d'impliquer de l'incertitude et des résistances par manque de compétences des personnels et par manque de justice organisationnelle pouvant prendre en charge ces évolutions (Steiner \& Rolland, 2006). Il s'en suit une accumulation de tensions au travail représentant un risque psychosocial certain. C'est pourquoi l'étude des conditions de travail des enseignants et de ses conséquences aussi bien sur l'individu que sur l'organisation s'avère particulièrement importante.

\section{Évolution des conditions de travail des enseignants}

Plusieurs recherches attestent de l'implication de facteurs reliés aux évolutions de l'environnement du travail dans la détérioration de la santé psychologique des enseignants et dans la genèse du stress professionnel (Demerouti, Bakker, Nachreiner \& Schaufeli, 2001; Janot-Bergugnat \& Rascle, 2008; Kyriacou, 2001; Perrenoud, 2010; Tardif \& Lessard, 1999; Vézina, 2003). Certains facteurs sont liés aux rôles et aux tâches de l'enseignement, d'autres sont davantage liés à l'environnement organisationnel du travail et aux objectifs pédagogiques spécifiques des établissements scolaires. En effet, dans le contexte de réforme, le rôle de l'enseignant évolue pour devenir un agent de développement qui contribue à asseoir les nouvelles orientations pédagogiques définies dans les réformes. En tant qu'expert du milieu scolaire, il participe à définir les besoins des élèves censés être au cœur de toute réforme. Son implication lui permet d'une part d'ajuster le contenu des réformes en fonction de la spécificité du milieu et d'autre part de répondre aux exigences d'une communauté circonscrite d'élèves. Sa tâche consiste à gérer les comportements des élèves en classe en les maintenant au travail pour assurer la transmission des savoirs, à éveiller leur curiosité, à développer un climat de classe positif (Debarbieux, Hamchaoui \& Moignard, 2013) et à participer à leur socialisation (Barrère, 2002). Tardif et Lessard (1999) le présentent comme une personne qui, tel un caméléon professionnel, doit assumer différents rôles en même temps. Il est à la fois psychologue, éducateur, parent, policier et confident, ce qui exige de lui des compétences complexes et multiples. De plus, avec l'évolution de la société et celle des nouvelles technologies de l'information et de la communication, le public scolaire a une connaissance de la technologie qui, le plus souvent, équivaut ou dépasse celle de l'enseignant. Les rapports qu'il entretient avec l'école sont également particuliers, ce qui amène l'enseignant, à 
travers les initiatives entreprises dans l'accomplissement de sa tâche, à adapter ses pratiques à la réalité de la classe (Pourtois \& Desmet, 2003). Or, les initiatives sont le plus souvent contrariées par la réalisation des prescriptions et se limitent à la gestion de la classe. Dès lors, affronter au quotidien ces tâches multiples crée chez l'enseignant un sentiment de dispersion et de débordement, ce qui est à l'origine de ses difficultés professionnelles et de sa souffrance au travail (Lantheaume, 2011). Il est évident que les transformations organisationnelles génèrent des contraintes professionnelles d'ordre psychosocial supplémentaires à celles du travail lui-même. En effet, les facteurs organisationnels, notamment la culture, le climat et le leadership, conditionnent les conduites au travail et jouent un rôle important pour la santé mentale (Brunet \& Savoie, 1999; Lison \& De Ketele, 2007). Ils peuvent avoir des incidences négatives sur la santé (Debarbieux, Hamchaoui \& Moignar, 2013). Un climat scolaire sain et un leadership positif de la direction sont les ingrédients de l'efficacité et du bien-être des enseignants (Curchod-Ruedi, Ramel, Bonvin, Albanese \& Doudin, 2013; Janosz, Thiebaud, Bouthillier \& Brunet, 2004). En revanche, un climat négatif, comme le souligne Horenstein (2006), peut être une source de stress et cause du présentéisme des enseignants ou d'absentéisme pour maladie. L'évaluation du climat devient indispensable pour prédire les risques psychosociaux et offrir en même temps des possibilités d'intervention en vue d'améliorer les conditions de l'environnement de travail (Gagnon, Paquet \& Courcy, 2008). En outre, la qualité des relations sociales contribue au développement d'un certain sentiment de sécurité et solidarité de la part de l'environnement de travail chez l'enseignant (Bakker, Demerouti \& Euwena, 2005; Moreau \& Mageau, 2013). Lorsque le soutien social fait défaut, des conflits s'élèvent, la communication se complique et l'enseignant se sent totalement isolé (Laugaa \& Bruchon-Schweitzer, 2005; Ponnelle, 2008; Rayou \& van Zanten, 2004). Cette situation est d'autant plus inconfortable qu'elle peut devenir aversive et induire du stress professionnel (Davezies, 2012; Gonik \& Kurt, 2004). En effet, la plupart des travaux développés dans le domaine de l'enseignement stipulent que les pressions liées aux caractéristiques du travail sont mises en cause dans les tensions psychologiques au travail (Hakanen, Bakker \& Schaufeli, 2006; Karasek \& Theorell, 1990).

\section{Le stress au travail chez les enseignants}

À l'instar des professions de service, l'enseignement est un secteur professionnel qui induit un haut niveau de stress (Cherniss, 1980; Kyriacou, 2001; Lantheaume, 2011; Janot-Bergugnat \& Rascle, 2008; Tardif \& Lessard, 1999). Le travail d'enseignant est miné par des facteurs psychosociaux. En témoignent certaines recherches qui mettent en évidence des sources de stress spécifiques au métier d'enseignant tels que la surcharge de travail, l'ambiguïté de rôle, l'iniquité, le manque de reconnaissance et la formation (Horenstein, 2006; Janot-Bergugnat 
\& Rascle, 2008; Kyriacou, 2001; Maroy, 2005). Le stress figure parmi les risques psychosociaux engendrés par les caractéristiques des conditions de travail (exigences, marge de manœuvre, contrôle sur la tâche, contenu, etc.), les facteurs organisationnels et relationnels (culture, climat, leadership, type de communication, etc.) ayant un impact sur la santé mentale et physique de l'individu. À ce propos, diverses études épidémiologiques ont apporté des précisions sur ces risques selon des remplacer par des modèles d'analyses différents, couplés à des outils de mesure (Derrienic \& Vézina, 2001). Le modèle Job Strain de l'astreinte professionnelle (Karasek, 1979) est largement utilisé dans les recherches scientifiques. Il permet de faire le lien entre le vécu du travail et les risques que ce travail fait courir à la santé mentale (stress, dépression et burnout) et physique (risque de maladies cardio-vasculaires). Il est construit sur la base de deux caractéristiques de la situation professionnelle: la demande psychologique (associée à la charge de travail) et la latitude décisionnelle (degré d'autonomie dans l'organisation des tâches, utilisation des compétences et participation aux décisions). Dans ce modèle, le stress résulterait de la combinaison de ces deux caractéristiques de la situation professionnelle. Leur croisement détermine le niveau de stress selon quatre types de situations de travail. La situation de travail, qui provoque un stress élevé "tension au travail» ou Job Strain, est celle qui résulte de la combinaison d'une demande psychologique élevée et d'une latitude décisionnelle faible, alors que la moins contraignante dite «détendue» est celle qui combine une demande faible et un niveau de latitude élevé. La situation alliant une forte demande et un niveau élevé de latitude (nommée «active») est favorable à la santé et propice au développement de nouveaux comportements, alors que celle qui allie un niveau faible aussi bien de demande que de latitude est appelée "passive». Ce modèle a été enrichi par un troisième facteur, «le soutien social», qui réfere au soutien organisationnel apporté par les collègues et la hiérarchie. Le soutien aurait un effet modérateur sur le stress, sa présence atténue les symptômes de tension travail et son absence l'accentue, générant un épuisement professionnel iso-strain (Karasek \& Theorell, 1990). Cette dernière situation a un effet pathogène et plusieurs études montrent la possibilité d'une évolution du stress chronique en burnout (Genoud, Brodard \& Reicherts, 2009). L'étude Euroteach (Verhoeven, Maes, Kraaij \& Joekes, 2003), menée sur 2796 enseignants du secondaire issus de treize pays européens, rapporte des liens significatifs entre le contrôle, le sentiment d'utilisation des compétences et l'épuisement professionnel. De nombreuses enquêtes nationales en France confortent ce constat. Une étude sur la qualité de vie au travail des enseignants (Horenstein, 2006) révèle que parmi les enseignants qui ont participé à l'enquête $(\mathrm{N}=2568), 36,42 \%$ jugent leur santé moyenne à mauvaise. Pour les enseignants du second degré, $43.94 \%$ signalent une insatisfaction importante au travail. Le quart des enseignants (24\%) du premier degré et le tiers (34\%) du second degré déclarent souffrir de difficultés physiques ou psychiques. Ils étaient absents en moyenne deux fois par an pour une durée de 11 jours pour des raisons de fatigue 
et de stress. L'enquête réalisée par Carrefour Santé Sociale (2011) sur 5000 enseignants a montré que $24 \%$ sont en état de stress permanent au travail et 14 $\%$ souffrent d'épuisement professionnel.

Les études évoquées mettent au jour l'influence des conditions de travail sur le stress des enseignants dans un contexte d'évolution du métier. Seulement, leur impact peut être différent en fonction des facteurs personnels, notamment le sexe (Bruchon-Schweitzer \& Dantzer, 1994). Ceci suppose la présence d'une variabilité concernant la vulnérabilité aux situations de travail. En effet, les femmes sont plus exposées à des tensions au travail que les hommes (Niedhammer, Chanstang, Gendrey, David \& Degioanni 2006). Elles déclarent manquer de latitude et de soutien social et subir des pressions (Niedhammer, David, Bugel \& Chea, 2001). Selon Fotinos et Horenstein (2011), les enseignantes sont vulnérables à l'épuisement émotionnel et à la perte du sentiment d'efficacité, tandis que les enseignants manifestent une attitude de repli sur soi.

Ainsi, ce stress lié à l'activité professionnelle des enseignants a été largement documenté par de nombreux travaux à l'échelle internationale; il a aussi fait l'objet de peu d'études en milieu tunisien (Chennoufi, Ellouze, Cherif, Mersni \& M'rad, 2012). C'est dans cette perspective que cette étude a été réalisée afin d'identifier les différentes contraintes rencontrées par les enseignants au travail et de repérer les facteurs stressants et, de là, prédire la présence du stress professionnel. En nous référant au modèle de Karasek (1979), nous postulons que les restrictions de l'autonomie et l'accroissement des exigences édictées par les conditions de travail favorisent l'émergence du stress professionnel chez les enseignants. Les facteurs stressants seraient liés à l'écart entre les ressources mises en œuvre et les spécificités de l'organisation du travail. À cet égard, on peut supposer que les caractéristiques spécifiques de l'organisation du travail à chaque type de collège pourraient conditionner le stress professionnel chez les enseignants.

\section{Problématique et hypothèse}

Dans le système éducatif tunisien, les établissements scolaires de l'enseignement secondaire sont classés en trois catégories: collèges d'enseignement général, collèges pilotes et collèges professionnels.

Les collèges pilotes sont des établissements élitistes dans lesquels sont inscrits uniquement les élèves les plus brillants, destinés à suivre un programme d'enseignement poussé. L'enseignement est assuré par des enseignants spécialisés ayant un niveau élevé de diplômes (maîtrise, master ${ }^{3}$ ) et dont l'expérience d'enseignement est bien reconnue. Le volume horaire est plus élevé que celui fixé dans les autres établissements. La qualité des conditions de travail des enseignants et d'apprentissage des élèves est de très loin supérieure à celle observée dans les autres établissements publics car ils bénéficient de ressources importantes 
allouées par l'État. Les collèges d'enseignement général sont destinés à des élèves de niveaux et de provenances hétérogènes. L'enseignement est assuré par des enseignants ayant des niveaux de formation variés (deux ans d'études supérieures, maîtrise, master). Certains ne sont pas titulaires de diplôme dans la matière qu'ils enseignent. Le volume horaire est identique dans tous les établissements. Les conditions de travail sont marquées par des ressources limitées (matériels, équipements, classes chargées, etc.). Dans les collèges professionnels, l'enseignement a pour but d'orienter les habiletés et les performances des élèves vers le travail pratique et de les préparer à la vie professionnelle. Cet enseignement est considéré comme un enseignement de rattrapage pour des élèves ayant mal réussi les études du cycle primaire d'enseignement. Les activités scolaires sont moins importantes en volume horaire que les apprentissages manuels. Les enseignants qui exercent dans ces établissements ont des niveaux de formations différents (diplôme professionnel, deux ans d'études supérieures, ou maîtrise).

Il est clair que ce système d'organisation scolaire présente des particularités liées aux objectifs pédagogiques spécifiques à chaque type de collège, à la diversité de la population scolaire et à l'organisation du travail. À ce sujet, en se référant au modèle de Cameron et Quinn (2006), Hafsi (2013) précise que les trois types de collèges ont une configuration de climat organisationnel spécifique. Dans les collèges d'enseignement général prédomine un climat de type Règle, alors qu'un climat de type Soutien dans les collèges professionnels. En revanche, dans les collèges pilotes, le climat est composite (Règle, Soutien, Innovation et Objectif).

Au vu de l'ensemble des caractéristiques spécifiques à chaque établissement et de leurs types de climat, nous avons émis les hypothèses suivantes:

1. Les enseignants des collèges d'enseignement général seraient plus stressés que leurs collègues des collèges pilotes et professionnels.

2. Les femmes seraient plus exposées au stress au travail que leurs collègues hommes.

3. Exercer dans un collège d'enseignement général augmenterait la probabilité de l'émergence du stress professionnel.

La principale variable dépendante dans notre étude correspond au stress professionnel. Nous utilisons deux variables indépendantes: sexe de l'enseignant et types de collèges. Cette dernière correspond aux spécificités organisationnelles directement liées au type de climat et aux conditions de travail qui prédominent dans le collège où l'enseignant évolue. 


\section{Méthodologie}

\section{Les participants}

Notre étude a été réalisée dans 51 collèges publics des cinq Délégations Régionales du Grand-Tunis, comprenant 42 collèges d'enseignement général, 3 collèges pilotes et 6 collèges professionnels. Sur les 600 questionnaires distribués aux enseignants, $351(58,5 \%)$ ont été complétés et remis. Le groupe de répondants se compose de 203 femmes (57.8\%) et 148 hommes (42.2\%). Sur les 351 enseignants, $83 \%(\mathrm{n}=291)$ exercent dans des collèges d'enseignement général, $12 \%(\mathrm{n}=41)$ dans des collèges professionnels et $5 \%(\mathrm{n}=19)$ dans des collèges pilotes.

\section{Instrument d'évaluation du stress au travail}

Le Job Content Questionnaire de Karasek figure parmi les questionnaires les plus utilisés sur le plan international pour mesurer l'exposition à des facteurs de risque de stress. Le choix du questionnaire se justifie, d'une part par le fait qu'il soit court et auto-administrable et d'autre part par sa validité approuvée dans plusieurs études (Larocque, Brisson \& Blanchette, 1998; Niedhammer et al., 2006). La version originale comporte 112 items, celle de l'enquête Sumer (20022009) est composée de 26 items (Niedhammer et al., 2006 ; Arnaudo, Léonard, Sandret, Cavet, Coutrot \& Rivalin, 2012) et celle de la cohorte de Gazel 1997 composée de 31 items (Niedhammer, 2002)

Dans la présente étude, la version utilisée, composée de 29 items, a été traduite en langue arabe. La cohérence interne basée sur le coefficient alpha de Cronbach est de 0.82 pour la latitude décisionnelle, 0.60 pour la demande psychologique et 0.91 pour le soutien social. Elle présente les qualités psychométriques requises (la moyenne sur l'ensemble des alphas de Cronbach est de 0.77). Une analyse factorielle avec rotation oblique (promax) a mis en évidence trois dimensions expliquant $86 \%$ de la variance expliquée.

Les sujets sont invités à se prononcer sur une échelle de Likert en quatre points: «Pas du tout d'accord», «Pas d'accord», «D'accord» et «Tout à fait d'accord» codés de 1 à 4 pour nuancer les réponses en fonction du degré d'affirmation jugé pertinent pour les propositions soumises aux participants:

- La demande psychologique ( 9 items) est liée aux caractéristiques de la tâche, aux exigences psychologiques du travail qui se traduisent par une quantité importante de travail, des demandes contradictoires, des interruptions successives et des contraintes de temps. Il s'agit de charge mentale et psychologique qui renvoie à la pression temporelle, à l'effort attentionnel nécessaire à la résolution de problèmes associée à ce travail.

- La latitude décisionnelle ou contrôle (9 items) est une dimension composée de deux sous-échelles: utilisation des compétences et qualifications pour être créatif et innovant, autorité décisionnelle. 
- Le soutien social (11 items) regroupe l'ensemble des interactions sociales, des aides et de la reconnaissance dont peut bénéficier le travailleur de la part de ses collègues et de ses supérieurs hiérarchiques. Il favorise l'installation d'un esprit d'équipe et d'un degré important de cohésion dans le groupe de travail. À partir des réponses, une partition au niveau des valeurs médianes (inférieures / supérieures à la médiane) de la latitude décisionnelle, de la demande psychologique et du soutien social est opérée. Sur la base des dimensions «latitude décisionnelle» et "demande psychologique» dichotomiques, les répondants sont classés selon une typologie de quatre situations de travail définie: "détendue», «active», "passive», «tendue». L'absence du soutien social devient une source supplémentaire de tension et entraine un état d'isolement au travail et d'épuisement professionnel.

Une variable binaire dite de stress a été construite au cours de l'analyse des données, la modalité stress correspondant à la «situation tendue» et la modalité «pas de stress» réunissant les trois autres modalités de situations.

\section{Procédure}

La procédure pour la collecte des données a été réalisée par questionnaire en langue arabe en format papier rempli par les enseignants. Après explication de l'objectif de l'étude, certains enseignants étaient coopératifs et ont accepté volontairement de remplir le questionnaire.

\section{Analyse des données}

Des analyses statistiques univariées ont été réalisées pour mettre en évidence les situations de travail perçues par les enseignants. Des tests de Khi2 ( $x 2)$ ont permis de mesurer la relation entre les différentes variables pour éprouver l'indépendance entre les composantes de stress professionnel et les types de collèges. Pour la comparaison des moyennes, des tests d'Anova ont été utilisés ainsi que des tests de Tukey-Kramer (post-hoc). Enfin, des analyses bivariées et prédictives par régressions logistiques ont été appliquées pour étudier la relation entre la variable explicative (types de collèges) et une variable expliquée (latitude faible).

\section{Résultats}

Par construction, le score de latitude décisionnelle peut prendre des valeurs allant de 24 à 96, la demande psychologique de 12 à 36 et le soutien social de 12 à 44. Les tests de Student n'ont pas permis de déceler une quelconque différence significative entre les scores moyens aux dimensions du JCQ des hommes et des femmes (Tableau 1). 
Tableau 1: Les scores globaux du JCQ des enseignants selon le sexe

\begin{tabular}{lcccccc}
\hline & \multicolumn{2}{c}{ Hommes $(\mathbf{n}=\mathbf{2 0 3})$} & \multicolumn{2}{c}{ Femmes $(\mathbf{n}=\mathbf{1 4 8})$} & $\mathbf{t}(\mathbf{3 4 9})$ & $\mathbf{p}$ \\
\cline { 2 - 7 } & $\mathbf{M}$ & $\mathbf{E T}$ & $\mathbf{M}$ & $\mathbf{E T}$ & & \\
\cline { 2 - 7 } Latitude décisionnelle & 59.2 & 14.4 & 60.9 & 16.0 & 1.01 & $0.28 \mathrm{~ns}$ \\
Demande psychologique & 22.6 & 3.8 & 22.7 & 4.2 & 0.21 & $0.83 \mathrm{~ns}$ \\
Soutien social & 27.1 & 7.2 & 28.2 & 7.6 & 1.33 & $0.19 \mathrm{~ns}$ \\
\hline
\end{tabular}

Note: ns (non significatif).

La moyenne pour la latitude est de $65.7(\sigma=13.6)$ dans les collèges professionnels, de 59,6 $(\sigma=14.8)$ dans les collèges d'enseignement général et 58,0 $(\sigma=23.3)$ dans les collèges pilotes. Pour la demande psychologique, la moyenne des scores dans les collèges pilotes est de $22.9(\sigma=4.5)$, celle des collèges d'enseignement général est de $22.7(\sigma=4.1)$ et celle des collèges professionnels est de $22.7(\sigma=$ 3.7). Concernant le soutien social, le score moyen dans les collèges professionnels est de $28.4(\sigma=7.6)$, de $27.7(\sigma=7.5)$ dans les collèges d'enseignement général et de $27.4(\sigma=7.7)$ dans les collèges pilotes (Tableau 2$)$.

Tableau 2: Les scores globaux du JCQ des enseignants selon le type de collège

\begin{tabular}{|c|c|c|c|c|c|c|c|c|c|c|c|c|c|c|c|}
\hline \multirow[b]{4}{*}{$\begin{array}{l}\text { Utilisation des } \\
\text { compétences }\end{array}$} & \multicolumn{6}{|c|}{ Moyenne } & \multicolumn{3}{|c|}{ Médiane } & \multicolumn{6}{|c|}{ Amplitude } \\
\hline & \multicolumn{2}{|c|}{$\begin{array}{c}\text { EG } \\
n=291\end{array}$} & \multicolumn{2}{|c|}{$\underset{n=19}{E P}$} & \multicolumn{2}{|c|}{$\begin{array}{l}\text { EPF } \\
n=41\end{array}$} & \multirow{2}{*}{$\begin{array}{c}\text { EG } \\
n= \\
291\end{array}$} & \multirow{2}{*}{$\begin{array}{l}\text { EP } \\
n= \\
19\end{array}$} & \multirow{2}{*}{$\begin{array}{c}\text { EPF } \\
n= \\
41\end{array}$} & \multicolumn{2}{|c|}{$\begin{array}{c}\text { EG } \\
\mathrm{n}=291\end{array}$} & \multicolumn{2}{|c|}{$\underset{n=19}{E P}$} & \multicolumn{2}{|c|}{$\begin{array}{c}\text { EPF } \\
n=41\end{array}$} \\
\hline & $\mathbf{M}$ & (ET) & $\mathbf{M}$ & $(\mathbf{E T})$ & $\mathbf{M}$ & (ET) & & & & Min & $\operatorname{Max}$ & Min & Max & Min & Max \\
\hline & 30.5 & ( 8.2) & 29.2 & (12.2) & 32.4 & $(7.8)$ & 30 & 26 & 32 & 12 & 48 & 12 & 48 & 16 & 48 \\
\hline $\begin{array}{l}\text { Autorité } \\
\text { décisionnelle }\end{array}$ & & (9.4) & 28.8 & (12.3) & 33.3 & (8.3) & 28 & 28 & 36 & 12 & 48 & 12 & 48 & 16 & 48 \\
\hline & & (14.8) & 58.0 & (23.3) & 65.8 & (13.6) & 60 & 58 & 68 & 24 & 96 & 24 & 96 & 36 & 96 \\
\hline & 2.7 & (4.1) & 22.9 & (4.5) & 22.7 & (3.7) & 23 & 23 & 22 & 12 & 36 & 15 & 33 & 16 & 31 \\
\hline & & $(4.0)$ & 14.4 & ( 4.8) & 16.0 & (3.9) & 15 & 14 & 17 & 6 & 24 & 7 & 23 & 6 & 23 \\
\hline & 12.5 & $(4.1)$ & 13.0 & (3.5) & 12.4 & (4.4) & 12 & 13 & 12 & 5 & 20 & 7 & 20 & 5 & 20 \\
\hline $\begin{array}{l}\text { Soutien } \\
\text { social }\end{array}$ & 27.7 & (7.5) & 27.4 & $(7.7)$ & 28.4 & (7.6) & 28 & 26 & 27 & 12 & 44 & 17 & 43 & 11 & 44 \\
\hline
\end{tabular}

Note: EG: Collège d'enseignement général, EP: Collège d'enseignement pilote, EPF: Collège d'enseignement professionnel.

Les analyses de variance mettent en évidence des différences significatives entre les trois types de collèges au niveau de la latitude décisionnelle $\mathrm{F}(2,348)=3.13$; $\mathrm{p}=0.04$. Des différences significatives ont été également observées au niveau de la sous-dimension autorité décisionnelle $\mathrm{F}(2,348)=3.51 ; \mathrm{p}<0.05)$. Les tests post-hoc de Tukey, appliqués pour comparer les trois types de collèges deux à deux, montrent que les collèges professionnels ont une moyenne de 
latitude décisionnelle $(\mathrm{p}=0.04)$ et d'autorité décisionnelle $(\mathrm{p}=0.02)$ significativement différentes de celles des collèges d'enseignement général. L'écart similaire observé entre les scores des collèges professionnels et les collèges pilotes n'est pas significatif du fait de la faiblesse de l'effectif et la grande dispersion des scores autour de la moyenne, dans les collèges pilotes. En revanche, il n'y a pas de différence significative entre les moyennes des collèges d'enseignement général et des collèges pilotes.

Pour identifier les caractéristiques des situations de travail telles qu'évaluées par les enseignants, les variables (demande psychologique, latitude décisionnelle et soutien social) ont été partitionnées au niveau des médianes des scores globaux comme indiqué dans le tableau 3.

Tableau 3: Médianes des scores globaux des dimensions du JCQ

\begin{tabular}{lccc}
\hline & Latitude décisionnelle & Demande psychologique & Soutien-Social \\
\hline Médiane & 60 & 23 & 28 \\
\hline
\end{tabular}

Les résultats obtenus permettent de constater que sur les 351 enseignants, environ $21 \%$ (34 femmes et 38 hommes) se situent dans le quadrant «détendus» (faible demande psychologique et forte latitude décisionnelle), 27\% (38 hommes et 58 femmes) dans le quadrant "passifs» faiblement chargé en demande psychologique et en latitude décisionnelle. Pour 34\% des enseignants ( 49 hommes et 72 femmes) l'environnement professionnel est perçu comme "actif», exigeant en demande psychologique avec des niveaux élevés d'attitude décisionnelle.

Presque un cinquième de l'échantillon (62 personnes) soit 17\% des femmes (35) et $18 \%$ des hommes (27) est en situation de tension psychologique. Ces derniers représentent un groupe à risque en termes de stress puisqu'ils se déclarent soumis à une forte demande psychologique associée à une faible latitude décisionnelle (Tableau 3). Au sein de ce groupe, les proportions de femmes $(56 \%)$ et d'hommes $(44 \%)$ sont similaires à celles de notre échantillon $\left(x^{2}(3)=1.20 ; p=0.75\right)$.

Tableau 4: Prévalence d'exposition au stress au travail

\begin{tabular}{ccccccc}
\hline & Actif & Détendu & Passif & Tendu (stress) & n & \% \\
\hline Femme & 72 & 38 & 58 & 35 & 203 & 57.8 \\
Homme & 49 & 34 & 38 & 27 & 148 & 42.2 \\
Total n & 121 & 72 & 96 & 62 & 351 & 100 \\
$\quad \%$ & $34 \%$ & $21 \%$ & $27 \%$ & $18 \%$ & & $100 \%$ \\
\hline
\end{tabular}

Lorsque l'on analyse le stress en fonction des types de collèges, les résultats montrent que parmi les enseignants des collèges d'enseignement général, 20\% $(\mathrm{n}=58)$ s'estiment stressés contre $11 \%(\mathrm{n}=2)$ de leurs collègues des collèges pilotes et $5 \%(\mathrm{n}=2)$ des collèges professionnels (Tableau 5). 
Tableau 5: Prévalence d'exposition au stress au travail dans les trois types de collège

\begin{tabular}{lcccccccccc}
\hline & \multicolumn{2}{c}{ Actif } & \multicolumn{2}{c}{ Détendu } & \multicolumn{2}{c}{ Passif } & \multicolumn{2}{c}{$\begin{array}{c}\text { Tendu } \\
\text { (stress) }\end{array}$} \\
\hline & $\mathbf{n}$ & $\%$ & $\mathbf{n}$ & $\%$ & $\mathbf{n}$ & $\%$ & $\mathbf{n}$ & $\%$ & $\%$ \\
Collège d'enseignement général & 96 & $33 \%$ & 58 & $20 \%$ & 79 & $27 \%$ & 58 & $20 \%$ & 100 \\
Collège pilote & 8 & $42 \%$ & 1 & $5 \%$ & 8 & $42 \%$ & 2 & $11 \%$ & 100 \\
Collège professionnel & 17 & $41 \%$ & 13 & $32 \%$ & 9 & $22 \%$ & 2 & $5 \%$ & 100 \\
\hline
\end{tabular}

Des différences significatives ont été observées entre les types de collèges et les différentes situations de travail $\left(X^{2}(6)=12.94 ; \mathrm{p}=0.04\right)$. Si l'on s'intéresse qu'aux deux groupes «tendus/non tendus (actifs, passifs, détendus)» et types de collèges, nous retrouvons le même lien $\left(X^{2}(2)=0.30 ; p=0.04\right)$. De plus, si l'on prend en compte le niveau (fort/faible) des deux principales composantes du stress au travail et si l'on examine les différences entre les types de collèges, les tests de $\mathrm{Khi}^{2}$ révèlent des liens significatifs entre types de collèges et latitude décisionnelle "forte/faible» $\left(X^{2}(2)=6.42 ; \mathrm{p}=0.04\right)$. En revanche, aucune différence significative n'a été observée entre types de collèges et demande psychologique (forte/faible) $\left(\mathrm{X}^{2}(2)=0.62 ; \mathrm{p}=0.73\right)$, de même que pour le soutien social $\left(X^{2}(2)=0.28 ; p=0.86\right)$. Nous remarquons qu'en grande majorité $(73 \%)$, les enseignants des collèges professionnels déclarent une forte latitude, un peu plus de la moitié $(53 \%)$ pour les enseignants des collèges d'enseignement général, et (47\%) pour ceux des collèges pilotes.

Pour caractériser ces différences, deux régressions logistiques ont été réalisées. La première modélise le risque d'exposition aux situations tendues (forte tension) par rapport aux autres situations (actives, passives, détendues: faible tension) par types de collèges. Le test du rapport de vraisemblance $\left(\mathrm{X}^{2}(2)=\right.$ 7.86; $\mathrm{p}=0.02$ ) indique que la perception du stress n'est pas la même selon le type d'enseignement. Le risque d'être exposé au stress dans les collèges pilotes n'apparait pas significativement différent de celui des collèges d'enseignement général (variable de référence). En revanche, ce risque est cinq fois moins important dans les collèges professionnels que dans les collèges d'enseignement général $(\mathrm{OR}=0.21$, significatif à $5 \%$ ) (Tableau 6).

Tableau 6: Régression logistique prédisant le stress dans les types de collège

\begin{tabular}{|c|c|c|c|}
\hline Test & Chi 2 & DF & $\mathbf{p}$ \\
\hline Ratio de vraisemblance & 7.86 & 2 & $0.02^{*}$ \\
\hline Score & 6.30 & 2 & $0.04^{*}$ \\
\hline Wald & 5.37 & 2 & 0.06 \\
\hline Effet & Odd ratio & $95 \%$ IC Wald & \\
\hline Collège pilote vs Collège d'enseignement général & 0.473 & $0.106-2.104$ & \\
\hline Collège professionnel vs Collège d'enseignement général & 0.206 & -0.878 & * \\
\hline
\end{tabular}

Note: ${ }^{*} \mathrm{p}<.05$. 
La seconde régression logistique modélise la latitude décisionnelle faible par rapport à forte, en fonction du type d'établissement scolaire. Le test du rapport de vraisemblance $\left(x^{2}(2)=6.70 ; p=0.03\right)$ est significatif indiquant qu'il existe bien un risque de stress différent selon les types de collèges. Un enseignant exerçant dans un collège d'enseignement général semble avoir environ deux fois et demi plus de risque de manquer de latitude décisionnelle qu'un collègue du collège professionnel $(\mathrm{OR}=0.41$, significatif à $5 \%)$. En prenant comme référence les collèges pilotes, le risque d'être privé de latitude décisionnelle est 3 fois plus important chez les enseignants des collèges pilotes que celui des enseignants des collèges professionnels $(\mathrm{OR}=3.0$, significatif à $5 \%)$. En revanche, ce risque n'est pas significativement différent pour les enseignants des collèges d'enseignement général et pilotes $(\mathrm{OR}=1.3$ non significatif à $5 \%)$ (Tableau 7).

Tableau 7: Régression logistique prédisant la latitude (faiblelforte) dans les types de collège

\begin{tabular}{llll}
\hline Test & Chi 2 & DF & p \\
\hline Ratio de vraisemblance & 6.70 & 2 & $0.03^{*}$ \\
Score & 6.42 & 2 & $0.04^{*}$ \\
Wald & 6.12 & 2 & $0.04^{*}$ \\
Effet & Odd ratio & $\mathbf{9 5} \%$ IC Wald & \\
\cline { 2 - 3 } Collège pilote vs Collège d'enseignement général & 1.249 & $0.493-3.164$ & \\
Collège professionnel vs Collège d'enseignement général & 0.412 & $0.199-0.854$ & $*$ \\
\hline
\end{tabular}

Note: ${ }^{*} \mathrm{p}<.05$.

En résumé, les enseignants des collèges d'enseignement professionnels apparaissent, dans notre étude, moins exposés aux risques psychosociaux. Ce qui les distingue des autres enseignants est précisément la latitude décisionnelle.

\section{Discussion}

L'objectif de notre recherche a visé l'identification du rôle du type de collège dans lequel les enseignants exercent dans la détermination du stress au travail. Les contraintes et les exigences fortes demandées à ces professeurs d'enseignement général pourraient occasionner une pression qui les amènerait à être plus stressés que leurs collègues des collèges d'enseignement pilote ou d'enseignement professionnel. Tout d'abord, les analyses des résultats mettent en évidence des différences de proportion de stress au travail chez les enseignants. Cela renvoie d'une part au rôle significatif des conditions de travail comme le soulignent les travaux de Karasek (1979), Niedhammer et al. (2001) et Gonik et Kurth (2004) et d'autre part à la signification attribuée aux stresseurs, modulée par certaines caractéristiques individuelles. 
Les enseignants des établissements d'enseignement général ressentent davantage de stress au travail que leurs collègues des collèges professionnels. Ils perçoivent leur travail comme ayant des exigences excessives qui dépassent leurs ressources. Cette différence peut être attribuable aux particularités liées aux objectifs pédagogiques spécifiques à ce type de collège, au type de climat organisationnel, à la diversité de la population scolaire et à l'organisation du travail (H1). L'effet de sexe (H2) apparaît peu important dans notre étude, bien que des travaux antérieurs aient mis en évidence des variabilités interindividuelles dans les réponses aux stresseurs (Fotinos \& Horenstein, 2011; Janot-Bergugnat $\&$ Rascle, 2008; Karasek, 1979; Niedhammer et al., 2001). Ainsi, nos hypothèses ne sont que partiellement confirmées.

Il ressort de nos résultats que le manque de latitude décisionnelle contribue à l'explication du stress éprouvé par les enseignants des collèges d'enseignement général. En revanche, ni les exigences de travail en termes de demande psychologique ni le soutien social n'apparaissent comme sources dans ce ressenti. Ces résultats vont dans le même sens que ceux de Gonik et Kurth (2004) et Chennoufi et al. (2012) qui montrent que l'autonomie octroyée à l'enseignant ne suit pas le rythme d'évolution des prescriptions. En effet, les enseignants, dans leur pratique professionnelle sont confrontés d'une part aux efforts cognitifs exigés par l'accomplissement de diverses tâches qui leur sont attribuées et d'autre part aux injonctions et aux réformes successives du système scolaire qui diminuent leur pouvoir de prise de décision (Lantheaume, 2011; Lantheaume \& Hélou, 2008; Perrenoud, 2010). Néanmoins, les enseignants des collèges professionnels apparaissent moins exposés au stress contrairement à leurs collègues des collèges d'enseignement général (H3) parce qu'ils bénéficient d'une forte latitude décisionnelle. Cette différence s'explique par la spécificité de leur enseignement qui repose sur l'apprentissage pratique des métiers impliquant un mode de fonctionnement encadré et réel. C'est un enseignement où prévalent l'appropriation d'un savoir-faire et d'un savoir-être constitutifs d'une identité professionnelle naissante, proche de la vie sociale. Dans ce contexte, la nature de l'apprentissage ainsi que le sens qui lui est attribué donne à l'enseignant le sentiment d'être utile.

Les résultats de notre étude montrent donc que le prédicteur potentiel de stress est le manque de latitude décisionnelle. Ils démontrent également que même si les enseignants des collèges d'enseignement général et des collèges pilotes en disposent, elle apparaît toutefois insuffisante dans la gestion des situations de travail, notamment au regard de ce qu'ils font en classe, des injonctions contradictoires et des attentes des élèves (Vézina, Bourbonnais, Marchand \& Arcand, 2010). Comme le montre la littérature internationale, l'impossibilité de prendre des initiatives dans le travail participe fortement à l'apparition du stress et à la perte du sens du travail (Chennoufi et al., 2012; Karasek \& Theorell, 1990; Lantheaume \& Hélou, 2008; Moreau \& Mageau, 2013). 


\section{Conclusion}

Cette recherche souligne le rôle de l'organisation et des stratégies pédagogiques des établissements scolaires sur le stress perçu par les enseignants. Le point de départ de notre étude était d'explorer les spécificités contextuelles du système éducatif et de son évolution pour retracer l'étendue des changements opérés et leurs conséquences sur la santé au travail. Il en ressort qu'à la suite des réformes successives du système éducatif, certains paramètres de l'organisation du travail sont mis en cause dans la genèse du stress professionnel. De plus, les transformations sociétales en Tunisie, les choix politiques instables et les mutations dans le monde du travail participent à l'apparition d'un état de stress professionnel des enseignants.

L'identification de certains aspects explicatifs des risques psychosociaux dans l'environnement scolaire pourrait fournir des pistes d'interventions préventives de la santé au travail. En ce sens, la dimension de contrôle et de marges de manœuvre revendiquées par les enseignants pourrait être appréhendée comme un plan d'action afin d'impliquer davantage les enseignants dans le processus pédagogique de telle sorte que l'environnement de travail devienne plus sain et favorable à la santé.

Aujourd'hui, le système éducatif tunisien est confronté aux défis de la qualité. L'apport de notre étude servira à accroître l'attention portée aux conditions de travail, à la qualité de vie des enseignants et aussi aux procédés de qualité de l'enseignement. Il serait également nécessaire dans les recherches futures de poursuivre les investigations auprès d'un effectif plus important d'enseignants des trois types de collège en incluant les caractéristiques sociodémographiques (âge, niveau d'étude, années d'expérience, etc.) et organisationnelles (environnement de travail, nombre d'élèves par classe, taille des établissements, etc.). L'évolution du nombre d'établissements pilotes nous permettrait également de mieux équilibrer la distribution des effectifs dans les différents types de collèges.

\section{Notes}

1 Faculté des Sciences Humaines et Sociales de Tunis - Département de psychologie,

2 CNAM - INETOP (Laboratoire CRTD)

3 Jusqu'en 2007, la maîtrise correspond à quatre ans d'études supérieures et le master à 6 ans. 


\section{Références bibliographiques}

Arnaudo, B., Léonard, M., Sandret, N., Cavet, M., Coutrot, T. \& Rivalin R. (2012). L'évolution des risques professionnels dans le secteur privé entre 1994 et 2010: premiers résultats de l'enquête SUMER. Dares Analyses, 23, mars.

Bakker, A., Demerouti, E. \& Euwema, M. (2005). Job resources buffer the impact of job demands on burnout. Journal of Occupational Health Psychology, 10, (2), 170-180.

Barrère, A. (2002). Les enseignants au travail. Paris: L'Harmattan.

Bouttemont,C. (2002). Le système éducatif tunisien. Revue international d'éducation de Sèvre, 29, 1-9. http://ries.revues.org/1928

Bruchon-Schweitzer, M. \& Dantzer, R. (1994). Introduction à la Psychologie de la Santé. Paris: PUF.

Brunet, L. \& Savoie, A. (1999). Le climat de travail: un levier pour le changement. Montréal: Éditions Logiques.

Cameron, K. \& Quinn, R. (2006). Diagnosing and changing organizational culture. Sans Francisco: Jossey-Bass.

Carrefour Santé Social (2011). Santé et travail: étude des risques psychosociaux, de l'épuisement professionnel et des troubles musculo-squelettiques. Paris: Direction de la Santé MGEN.

Chennoufi, L., Ellouze, F., Cherif, W., Mersni, M. \& M'rad, M. F. (2012). Épuisement professionnel des enseignants tunisiens. L'Encéphale, 38, 480-487.

Cherniss, C. (1980). Professional burnout in human service organizations. New York: Praeger.

Curchod-Ruedi, D., Ramel, S., Bonvin, P., Albanese, O. \& Doudin, P.-A. (2013). De l'intégration à l'inclusion scolaire: implication des enseignants et importance du soutien social. ALTER, European Journal of Disability Research, 7, 135-147.

Davezies, Ph. (2012). Enjeux, difficultés et modalités de l'expression sur le travail: point de vue de la clinique médicale du travail. Perspectives interdisciplinaires sur le travail et la santé, 14, (2), 2-18.

Debarbieux, E., Hamchaoui, K. \& Moignar, D. B. (2013). Enquête de victimisation auprès des enseignants du second degré. FAS-USU, MEN. Consulté le 8 mars dans http:// www.academia.edu/11742893/ENQUE_TE_DE_VICTIMATION_ET_CLIMAT_ SCOLAIRE_AUPRE_S_DES_PERSONNELS_DU_SECOND_DEGRE

Demerouti, E., Baker, A.B., Nachreiner, F. \& Schaufeli, W.B. (2001).The Job demands-resources model : challenges for future research. South African Jounal of Industrial Psyhology, 37, (2), 1-9.

Derriennic, F. \& Vézina, M. (2001). Organisation du travail et santé mentale: approches épidémiologiques. Travailler, 5, 7-22.

Diet, E. (2001). Paradoxalité dans la formation et souffrance de l'identité professionnelle. Connexion, 75, 13-28.

Ennaifar, M. (2009). Mobilisation des acteurs et conduite de la réforme. In V. Tehio (Éd.), Actes du séminaire final de l'étude sur les réformes curriculaires (pp.99-115). Paris: Centre international d'études pédagogiques.

Fotinos, G. \& Horenstein, J. M. (2011). La qualité de vie au travail dans les lycées et collèges. Le «burnout des enseignants». Paris: MGEN.

Gagnon, S., Paquet, M. \& Courcy, G. (2008). Climat de travail et santé physique et psychologique. Actes du Congrès de l'Association internationale de psychologie du travail de langue française, août 2008, Québec, Canada.

Genoud, P.A., Brodard, F. \& Reicherts, M. (2009). Facteurs de stress et burnout chez les enseignants de l'école primaire. Revue européenne appliquée, 59, 37-45.

Gonik, V. \& Kurt, S. (2004). La santé psychosociale des enseignants et des enseignantes. Canada: Presse universitaire du Québec.

Hafsi, A. (2013). Condition de travail, qualité de vie et santé psychologique chez les enseignants des collèges du Grand-Tunis (thèse de doctorat non publiée). Tunis: Facultés des Sciences Humaines et Sociales de Tunis et Cnam-Paris. 
Hakanen, J. J., Bakker, A. B. \& Schaufeli, W. B. (2006). Burnout and work engagement among teachers. Journal of School Psychology, 43, 495-513.

Horenstein, M. (2006). La qualité de vie au travail des enseignants. Paris: MGEN.

Janosz, M., Thiebaud, M., Bouthillier, C. \& Brunet, L. (2004). Perception du climat scolaire et épuisement professionnel chez les enseignants, $13^{e}$ Congrès de psychologie du travail et des organisations AIPTLF. Bologne.

Janot-Bergugnat, L. \& Rascle, N. (2008). Le stress des enseignants. Paris: Armand Colin.

Karasek, R. (1979). Job demands, job decision latitude, and mental strain: implications for job redesign. Administrative Science Quarterly, 24, 285-308.

Karasek, R. \& Theorell, T. (1990). Healthy work; stress, productivity and the reconstruction of working life. New York: Wiley and Sons.

Kyriacou, C. (2001). Teacher stress: Directions for future research. Educationnal Review, 53, 28-35.

Lantheaume, F. (2011). La souffrance des enseignants, épiphénomène ou analyseur du métier et du système? In C. Meyor, La souffrance à l'école, Les Collectifs du Cirp (Cercle interdisciplinaire de recherches phénoménologiques) (pp. 6-18). Montréal: Université du Québec.

Lantheaume, F. \& Hélou Ch. (2008). La souffrance des enseignants. Une sociologie pragmatique du travail enseignant. Paris: PUF.

Lapointe, D. Boudrias, J. S. \& Brunet, L. (2011). Vérification de l'effet modérateur des ressources sur la relation entre les demandes et la tension psychologique au travail. In P. Desrumeaux, A. M. Vonthron, \& S. Pohl (Éd.), Qualité de vie, risque et santé au travail. Paris: L'Harmattan.

Larocque B., Brisson, C. \& Blanchette C. (1998). Internal Consistency, Factorial Validity and Discriminant Validity of the French Version of the Psychological Demands and Decision Latitude Scales of the Karasek Job Content Questionnaire. Rev. Epidemiol. Sante publique, 46, (5), 371-381.

Laugaa, D. \& Bruchon-Schweitzer, M. L. (2005). L'ajustement au stress professionnel chez les enseignants français du premier degré. L'orientation scolaire et professionnelle, 34, 499-519.

Lison, C. \& De Ketele, J. M. (2007). De la satisfaction au moral professionnel des enseignants: étude de quelques déterminants. Revue des sciences de l'éducation, 1, (33), 179-207.

Loi $\mathrm{n}^{\circ}$ 91-65 du 29 juillet 1991 relative au système éducatif. Journal Officiel de la République Tunisienne, $n^{\circ} 55$.

Loi $\mathrm{n}^{\circ} 2008-9$ du 11 février 2008, modifiant et complétant la loi d'orientation $\mathrm{n}^{\circ} 2002-80$ du 23 juillet 2002, relative à l'éducation et à l'enseignement scolaire. Journal Officiel de la République Tunisienne, $n^{\circ} 14$.

Maroy, C. (2005). Les évolutions du travail enseignant en Europe. Facteurs de changement, incidences et résistances. Les Cahiers de recherche en éducation et formation, $n^{\circ} 45$.

Moreau, E. \& Mageau, G.A. (2013). Conséquences et corrélats associés au soutien de l'autonomie dans divers domaines de vie. Psychologie française, 58, 195-227.

Niedhammer, I., Chanstang, J.F., Gendrey, L., David, S. \& Degioanni, S. (2006). Propriétés psychométriques de la version française des échelles de la demande psychologique, de la latitude décisionnelle et du soutien social du «Job Content Questionnaire» de Karasek: résultats de l'enquête nationale SUMER. Santé publique, 18, (3), 413-427.

Niedhammer, I., David, S., Bugel, I. \& Chea, M. (2001). Catégories socioprofessionnelles et exposition aux facteurs psychosociaux au travail dans une cohorte professionnelle. Travailler, 5, 23-45.

Niedhammer, I. (2002). Psychometric properties of the french version of the karasek job content questionnaire: a study of the scales of decision latitude, psychological demands, social support, and physical demands in the Gazel cohort. International Archives of Occupational and Environmental Health, 75, (3), 129-144. 
Perrenoud, P. (2010). Les processus de (dé)professionnalisation entre savoir, rapport au savoir et contrôle. Recherche en Éducation (CREN, Nantes), 8, 121-128.

Ponnelle, S. (2008). Contribution des déterminants personnels, organisationnels et des styles d'ajustement au stress dans l'explication de la santé subjective des enseignants du secondaire. L'orientation scolaire et professionnelle, 37, (2), 183-213.

Pourtois, J.P. \& Desmet, H. (2003). Vers une parentalité psychologique et pédagogique. Actes des Journées Nationales de la Formation SESSAD, Palais des Congrès, Reims, novembre 2002.

Rayou, P. \& van Zanten, A. (2004). Enquête sur les nouveaux enseignants. Changeront-ils l'école? Paris: Bayard.

Steiner, D. D. \& Rolland, F. (2006). Comment réussir l'introduction de changements: les apports de la justice organisationnelle. In C. Levy-Leboyer, C. Louche, \& J.P. Rolland (Éd.), RH: Les Apports de la Psychologie du Travail. 2. Management des Organisations (pp. 53-69). Paris: Éditions d'organisation.

SUMER (2002 et 2009)

Tardif, M. \& Lessard, C. (1999). Le travail enseignant au quotidien. Bruxelles: De Boeck Université.

Verhoeven, C., Maes, S., Kraaij, V. \& Joekes, K. (2003). The job demand- control- social support model and wellness/health outcomes: A European Study. Special Issue of Psychology and Health, 18, (4), 421-440.

Vézina, M. (2003). Stress au travail et santé psychique: rappel des différentes approches. In M. Néboit \& M. Vézina. (Éd.), Stress au travail et santé psychique (pp.47-58). Toulouse: Octarès.

Vézina, M., Bourbonnais, R., Marchand, A. \& Arcand., R. (2010). L'autoévaluation du stress au travail: un indicateur de santé trompeur. Perspectives interdisciplinaires sur le travail et la santé. Consulté le 5 mai 2013 dans http:// pistes.revues.org/2503

Mots-clés: Stress au travail, enseignement secondaire, enseignants

\section{Stress bei der Arbeit unter Lehrpersonen der Collèges in Tunesien}

\section{Zusammenfassung}

Der Zweck dieser Studie ist es, stresserzeugende Situationen unter Lehrpersonen der Sekundarstufe zu identifizieren. Der theoretische Rahmen stellt psychologische Stressmodelle im professionellen Umfeld bereit, im Besonderen das von Karasek. Wir vermuten, dass die Organisation und Verwaltung an Schulen beruflichen Stress auslösen. Wir haben diese Hypothese durch die JCQ-Umfrage an über 351 Lehrpersonen getestet. Die Umfrage wurde an drei Arten von weiterführenden Schulen vorgenommen. Die Ergebnisse zeigen, dass 18\% der Befragten mit Stresssituationen konfrontiert werden. Lehrpersonen von fachlichen und technischen Schulen sind weniger Stress belastet als die Anderen. Dieses erklärt sich durch mehr Entscheidungsspielraum und besseren Möglichkeiten zur Kontrolle.

Schlagworte: Arbeitsbedingter Stress, weiterführende Schulen, Lehrpersonen 


\section{Lo stress lavorativo degli insegnanti di scuole medie in Tunisia}

\section{Riassunto}

L'obiettivo dello studio è identificare le situazioni di lavoro che generano più stress professionale per gli insegnanti di scuola media in Tunisia. I riferimenti teorici trattano i modelli di stress psicologico nell'ambito professionale, in particolare quello di Karasek. Abbiamo ipotizzato che le condizioni di lavoro degli insegnanti nelle scuole medie d'istruzione generale generino stress professionale. Questa ipotesi è stata dimostrata con l'uso del test JCQ Karasek presso 351 insegnanti in tre scuole medie. I resultati mostrano che il $18 \%$ di loro si sentono stressati. Tuttavia gli insegnanti delle scuole medie professionali sono meno esposti allo stress rispetto agli altri perché hanno una forte autonomia decisionale e hanno un certo controllo sull'espletamento delle loro funzioni.

Parole chiave: Stress lavorativo, scuola secondaria, insegnante, professore/ professoressa.

\section{Occupational stress among secondary school teachers in Tunisia}

\section{Abstract}

The purpose of this study is to identify stress-generating situations among secondary school teachers. The theorical framework summons up models of psychological stress at work, particularly the Karasek's one. We hypothesize that organization and management of the schools induce occupational stress. We tested this hypothesis by conducting the JCQ survey on 351 teachers working in the three types of secondary schools. The results shown that $18 \%$ of the population express facing stressful situations. Teachers of professional and technical schools are less facing stress pressure than the others. They declare having more decisional latitude and control opportunities.

Keywords: Occupational stress, secondary schools, teachers. 